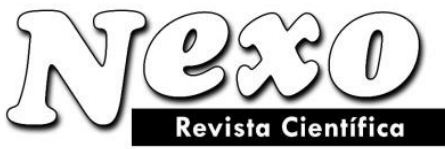

ISSN-E 1995-9516

Universidad Nacional de Ingeniería COPYRIGHT @ (UNI). TODOS LOS DERECHOS RESERVADOS

http://revistas.uni.edu.ni/index.php/Nexo

https://doi.org/10.5377/nexo.v34i06.13118

Vol. 36, No. 06, pp. 1575-1588/Diciembre 2021

(c) (i) $\odot$

\title{
Confluencia entre los ecosistemas forestales y el conocimiento científico frente al cambio climático: Marco legal peruano
}

\section{Confluence between forest ecosystems and scientific knowledge against climate change: Peruvian legal framework}

\author{
Jeanpierre Rodríguez Mirano* (D), Jacinto Joaquín Vértiz-Osores \\ Universidad Nacional Tecnológica de Lima Sur. Lima, Perú. \\ *Corresponding author E-mail: 2014100425@untels.edu.pe
}

(recibido/received: 25-julio-2021; aceptado/accepted: 15-septiembre-2021)

\begin{abstract}
RESUMEN
El presente artículo consta de un estudio exploratorio que reflexiona sobre el alcance de las políticas en cambio climático del Perú, la generación de conocimiento científico a partir del estudio de ecosistemas forestales y la factibilidad de un escenario de confluencia entre ambos. Se basó en un análisis cualitativo, a través del diseño de Teoría Fundada Emergente, de tipo Jurídico-comparativo. Se explicó el valor de la vulnerabilidad de un ecosistema forestal como herramienta de análisis de políticas de adaptabilidad frente al cambio climático, además de los beneficios instantáneos que devienen de la sistematización de información científica para facilitar el proceso de definición de estrategias hacia estas políticas.
\end{abstract}

Palabras claves: Cambio climático; Ecosistema; Política ambiental.

\begin{abstract}
This article consists of an exploratory study that reflects on the scope of climate change policies in Peru, the generation of scientific knowledge from the study of forest ecosystems and the feasibility of a scenario of confluence between both. It was based on a qualitative analysis, through the design of Emergent Founded Theory, of a juridical-comparative type. The value of the vulnerability of a forest ecosystem as a policy analysis tool for adaptation to climate change was explained, in addition to the instantaneous benefits resulting from the systematization of scientific information to facilitate the process of defining strategies towards these policies.
\end{abstract}

Keywords: Climate change; Ecosystem; Environmental policy.

\section{INTRODUCCION}

Adaptarse al cambio climático ante el momento incierto de los impactos significa que se debe tener un conjunto de opciones disponibles (Sung and Kim, 2016). Es de alta prioridad hacer frente y adaptarse a las perturbaciones forestales mientras se mantiene la biodiversidad y la resiliencia de los ecosistemas forestales (Dreyfus, 2015; Yousefpour et al., 2017). El presente trabajo discute las actuales políticas nacionales que responden al cambio climático, analiza la efectividad de la generación de conocimiento científico a partir 
del estudio de ecosistemas forestales y reflexiona sobre la factibilidad de un escenario de confluencia de ambas dentro del marco legal peruano.

Para el Perú, constituye un desafío la generación de datos e información con elevados niveles de rigor científico que permitan la compresión de la temática asociada a los cambios del clima y sus impactos. Además que posibilite la proyección y evolución de dichos cambios y efectos en el futuro, proporcione herramientas e insumos técnicos a investigadores, profesionales y académicos dedicados al tratamiento del problema y al planteamiento de las respectivas soluciones, con la finalidad de fortalecer las perspectivas de gestión en la toma de decisiones (Cadilhac et al., 2017). Actualmente se advierte un vacío en la generación de métricas que apoyen la preparación de análisis técnicos sobre mitigación y adaptación al cambio climático, así como el registro de las diferentes investigaciones que se realizan en el territorio nacional (Ministerio del Ambiente, 2016).

Frente a este panorama, es predecible que en el Perú los cambios del clima provoquen drásticas alteraciones en el equilibrio de los bosques, aumento de plagas y patógenos, cambios en los ciclos de evapotranspiración de la vegetación, mayor presencia de sequías e inundaciones, incremento en el nivel de sedimentos, incendios forestales, mortalidad de árboles e invasión de pasturas, entre otros, lo cual muestra la peculiaridad de los ecosistemas forestales hacia la alta vulnerabilidad (Organización de las Naciones Unidas para la Agricultura y la Alimentación, 2016, pág. 24).

En el marco de estos sucesos, se desprende que es necesario desarrollar un diálogo interinstitucional de alcance nacional que concluya en un consenso en torno a la necesidad de fortalecer los ecosistemas forestales como espacios de encuentro y coordinación de las entidades gubernamentales con el sector académico, que potencien ejes transversales como: capacidades técnicas e institucionales en cambio climático, comunicación, difusión, accesibilidad e intercambio de criterios sobre el conocimiento científico ya existente, y el uso de herramientas tecnológicas para recopilar, estructurar e integrar en un repositorio los estudios desarrollados en este campo temático.

\section{MÉTODOS}

Basado en un diseño de teoría fundada emergente (Hernández-Sampieri and Mendoza 2018), se pretende trascender al sujeto social para explicar y comprender fenómenos sociales más complejos, brindando un panorama particular del aprovechamiento de los ecosistemas forestales para conocimiento científico. Una iniciativa de esta naturaleza es de tipo jurídico-comparativo ya que establece las semejanzas y/o diferencias entre instituciones jurídicas o sistemas jurídicos, destacando los principales tratados, documentos, declaraciones e informes, así como la comprensión de las posibilidades de una protección amplia y holística de los ecosistemas forestales basada en el marco legal nacional existente (Bustamante 2009).

Esta propuesta se basa en un análisis cualitativo realizado entre los meses de junio y noviembre del 2020. Con énfasis específico en los desafíos y posibilidades enmarcados en el contexto de los imperativos impuestos por el cambio climático, en cuanto al desarrollo de una política de estrategias de adaptación al mismo, basado en conocimiento científico y en su sistematización, encontrándose en el orden de la construcción sistemática de los conceptos (Strauss and Corbin, 2002).

\section{RESULTADOS}

1. Alcance de las políticas en cambio climático en el Perú

Las continuas conexiones entre economía internacional y los recursos naturales se explican en la adopción de regulaciones ambientales, en los flujos de comercio internacional y en la formulación de la política comercial (Quijandría, 1993). A pesar de que el uso de incentivos económicos usualmente es más eficiente, 
en términos de costos para reducir los efectos del cambio climático, el enfoque predominante es el de fijación de estándares (Pasco-Font and Montoya, 1993). Esta dependencia tiene implicancias sobre el medio ambiente y las migraciones interregionales, como el caso de la economía cocalera en el Perú, situación que podría replicarse de continuar careciendo de enfoques adaptativos (Montero, 1995).

La estimación del valor de los servicios ambientales representa un elemento crucial en el proceso de toma de decisiones sobre los programas y las políticas ambientales por desarrollar, así como un instrumento de orientación para la implementación de patrones de producción y consumo más sostenibles. Lamentablemente, la determinación del valor de bienes ambientales, suelen estar basados principalmente en dos características metodológicas relacionadas a valores netamente económicos. La primera es si los datos de las observaciones provienen de razones que responden a preguntas hipotéticas de la forma de lo que se está dispuesto a pagar. La segunda es si el método genera un valor económico directamente o si este valor debe ser inferido de alguna técnica indirecta basada en un modelo de elección y comportamiento particular (Roa et al., 2004).

La adaptación de la sociedad al cambio climático requiere medidas que simultáneamente reduzcan la pobreza, protejan o restauren la biodiversidad y los servicios de los ecosistemas (Abrahams and Carr, 2017; Castells-Quintana et al., 2018). La adaptación basada en ecosistemas al cambio climático es aquella que tiene como objetivo combinar estos resultados y es particularmente relevante para las naciones en desarrollo que protegen la mayor parte de la biodiversidad planetaria y los ecosistemas saludables (Scarano, 2017). Frente a esto, el enfoque Ecosystem Values Assessment and Accounting (EVAA) se desarrolló para ayudar a informar de las posibles acciones de adaptación en respuesta a un clima cambiante. La EVAA combina múltiples modelos cuantitativos y la obtención de expertos de científicos y administradores de tierras. (Mercer et al., 2012).

Estos conceptos están estrechamente relacionados con la evaluación ambiental estratégica (EAE), que es una herramienta aplicada para hacer frente a la inclusión de los impactos ambientales en el ámbito estratégico en un momento oportuno cuando todavía se puede influir en la toma de decisiones, la EAE reconoce factores críticos que puede ser mayor al ámbito de análisis de los impactos ambientales. Lo importante es que al igual que la EVAA, forma parte del proceso de planificación en el que se considera oportunamente la evaluación ambiental estratégica como posibilidad para fortalecer los procesos de planificación ambiental en los diversos sectores, como un proceso voluntario que genere aceptación por parte de los emprendedores de proyectos, planes, programas y políticas de desarrollo del sector privado y público, y visto más como una oportunidad que como un obstáculo. (Flores 2005)

El Informe del Intergovernmental Panel on Climate Change (IPCC) $1.5^{\circ} \mathrm{C}$ enumera la identificación de las necesidades de capacidad local como clave para permitir que la gobernanza multinivel responda de manera efectiva al cambio climático. Los ecosistemas forestales, por su condición de vulnerabilidad, se ven desproporcionadamente afectados por el cambio climático, principalmente debido a su exposición a los impactos, así como a diversas interrupciones al sistema natural por actividades antropogénicas (IPCC, 2014). Un caso particular del desarrollo de un sistema integrado es el de Beijing, este proyecto respondió a la recomendación hecha por el quinto Informe de Evaluación del IPCC sobre el establecimiento de un estándar para medir y reportar el riesgo y la vulnerabilidad climáticos. Ejemplificó la evaluación de la vulnerabilidad urbana al cambio climático mediante el desarrollo de un enfoque integrado, cuyo principal resultado fue proporcionar un índice de vulnerabilidad integrado para todo Beijing (Zhang et al., 2019).

En el Perú, la Estrategia Nacional ante el Cambio Climático (ENCC) (Ministerio del Ambiente, 2015) considera que el principal reto asociado al cambio climático, es reducir los riesgos e impactos previsibles, fortaleciendo capacidades para enfrentarlos a través de una gestión integrada de los tres niveles de gobierno (Legislativo, Ejecutivo y Judicial) que permita reducir vulnerabilidades y aprovechar oportunidades. Así, frente al imperativo de responder a una integración de los lineamiento que permitan fortalecer el nivel de 
conocimiento científico y técnico, en el 2015 se actualizó la ENCC con los numerosos estudios sobre la materia bajo el trazo del objetivo de que las instituciones gubernamentales y privadas - en sus diferentes ámbitos - cuenten con conocimiento científico y técnico para una respuesta oportuna y efectiva frente al cambio climático (Ministerio del Ambiente, 2015)

Para lo mismo, la ENCC (Ministerio del Ambiente, 2015) especifica 37 medios de implementación, doce enmarcados en el subcapítulo de Conocimiento científico y tecnología, de los que se rescatan solo tres con vínculo directo a esta investigación, es decir, que proponen el conocimiento científico y su sistematización como guía que vincule actividades de adaptación al cambio climático. Estos son: (1) impulsar y fortalecer el desarrollo, mejoramiento y ampliación de sistemas nacionales y regionales de observación del clima, (2) crear líneas de investigación científica en el marco de la Agenda de Investigación Científica en Cambio Climático, y (3) diseñar y difundir herramientas e instrumentos de medición convencionales y no convencionales para la evaluación de la vulnerabilidad ante el cambio climático.

Estos medios de implementación, dado que no se explican a lo largo del documento, no describen herramientas ni instrumentos para su ejecución, no colindan acciones con otros medios, ni encuadran sus esfuerzos en el marco de políticas legales del Estado; se podría decir que carecen justamente de los recursos sobre los que este estudio reflexiona, no se ha evidenciado hasta ahora una operativización que brinde resultados tangibles.

En cuanto al fomento de una acción climática coordinada, el Estado peruano creó mediante Resolución Suprema Nº 005-2016-MINAM, el Grupo de Trabajo Multisectorial (GTM) (2018a) de naturaleza temporal, encargado de generar información técnica para orientar la implementación de las Contribuciones Nacionalmente Determinadas (CND). El GTM incluyó el área temática "bosques" dentro de las CND en adaptación al cambio climático, realzando la importancia de la conservación de los bosques en base a tres características: (1) su vulnerabilidad frente al cambio climático, (2) la disponibilidad de información de base $y$, (3) la existencia de una entidad responsable del cumplimiento de las metas, proponiendo finalmente al Servicio Nacional Forestal y de Fauna Silvestre.

Esto ha representado hasta entonces un hito en la gestión integral frente al cambio climático en el Perú, ello porque permitió sentar las bases para el desarrollo sostenible bajo en carbono y resiliente al clima y, porque el proceso de su implementación requirió el compromiso y la participación de todos los actores gubernamentales y no gubernamentales de la sociedad peruana. Las tres características descritas son sinérgicas con las áreas temáticas analizadas en esta investigación, pero difieren en cuanto a su comprensión y a la forma en que se pretende su implementación y uso.

En el primer producto del GTM (2018a), únicamente la tercera medida de adaptación 'Implementación de un programa nacional de monitoreo de la dinámica del bosque para medir el impacto del cambio climático y adaptarse a sus efectos' parece responder a la formulación de estrategias en base a conocimiento científico, en la que proponen el establecimiento de una red de parcelas permanentes de vegetación en 12 Áreas Naturales Protegidas en el bioma amazónico y el recojo de data histórica a partir de 1970. Pudiendo formularse bajo una alianza estratégica de correlación de datos actuales e históricos del clima que proporcione el Servicio Nacional de Meteorología e Hidrología y el Instituto Geofísico del Perú, con el fin de evaluar los cambios en los bosques producidos por el impacto del cambio climático.

Finalmente, limitan los logros de ésta y sus demás propuestas al indicador de 'Porcentaje de implementación de un programa nacional de monitoreo de la dinámica del bosque para medir el impacto del cambio climático y a partir de ello adoptar medidas de adaptación' recalcando que se desarrollaría en el ámbito nacional de las ANP. El porcentaje de cumplimiento que proyectan para el 2021 es de 15\%, esperándose alcanzar el logro de la meta en su totalidad para el 2030. 


\section{Generación de conocimiento científico a partir de ecosistemas forestales}

Los proyectos ambientales son una buena aproximación a la importancia que los distintos niveles de gobierno le dan a la toma de decisiones sobre la base de metodologías enmarcadas en estudios científicos, ya que su implementación es una manera de asignar las responsabilidades que tienen por ley. Las restricciones que enfrentan estos proyectos podrían devenir de la metodología en la que se basa su desarrollo, ejecución y posterior evaluación, además del nivel de gobierno que aprueba los estudios, entre otras variables de análisis. Los proyectos ambientales son los menos priorizados por los gobiernos subnacionales, algunos factores que podrían explicar esta diferencia tienen que ver con las dificultades metodológicas asociadas a estos proyectos (Kámiche and Cárdenas 2008).

Un ejemplo del uso excesivamente politizado de herramientas de política que contribuyen a escenarios poco sostenibles podría ser lo ocurrido de 1935 hasta 1990 respecto al desarrollo energético del Perú basado en un aparente escenario de explotación del petróleo y en la falta de compromiso del Estado para explotar el gas natural. No obstante, la aprobación de una nueva legislación modernizadora que respondía a criterios técnico científicos en la década de 1990, estableció un nuevo impulso de crecimiento en dicho sector (Wise, 2005). Este fenómeno se repitió en América Latina entre los años 1995-2007 con el propósito de aportar a la orientación de políticas públicas incentivos a la producción energética. Para lograr este objetivo, se identificó la mejor estimación del fenómeno en base a conocimiento científico, lo que permitió orientar las políticas públicas al incremento de mayor producción energética. Esto tras un modelado de las políticas públicas de trece países latinoamericanos efectuado por Dalmazzo-Bermejo et al. (2017).

Antes de definir las estrategias para la Gestión del Conocimiento, es importante identificar el conocimiento existente, quién lo posee, dónde se encuentra almacenado y cómo fluye entre quienes lo generan (BarceloValenzuela et al., 2008). Si bien la ciencia es importante para la gestión ambiental, crear una ciencia que sea creíble, destacada para los tomadores de decisiones y considerada legítima por los interesados es un desafío, el modelado colaborativo es un enfoque cada vez más utilizado para permitir una toma de decisiones eficaz basada en la ciencia (Ulibarri, 2018). En el campo de la investigación científica, es menester establecer las prioridades de investigación que se requiere o pretende conseguir a nivel de región y de país (Quintero et al. 2008). Para lo mismo se precisa que se aúnan estas investigaciones a un objetivo en común, al igual que sistematizar dicha información.

Para las instituciones con énfasis en la investigación es necesario incluir la gestión del conocimiento científico como un enfoque estratégico para marcar la diferencia, generar ventajas competitivas y mejorar su posición en su campo de especialización y en el entorno en que operan; se requieren ciertas condiciones previas para facilitar el proceso de definición de una estrategia (Beier et al., 2017). De hecho, las organizaciones deben conocer la evolución de su ambiente y elaborar instrumentos de gestión más eficaces, que les permita medir sus conocimientos, gestionarlos y convertirlos en valor (Ortiz and Ortega 2016).

El conocimiento científico por sí solo es insuficiente para modificar hábitos o impulsar reformas, más allá de crear conocimiento, la tecnología implica negociación, política y relaciones sociales. Las negativas a aceptar cambios, aun frente a la posibilidad del desastre natural, están asociadas a las explicaciones del origen de tales desastres, las cuales no siempre concuerdan con la explicación científica de la catástrofe (Lossio, 2015). Para que proporcione mejoras sustanciales, el conocimiento debe ser correctamente gestionado, de ahí que la clave esté en la Gestión del Conocimiento, como disciplina imprescindible en las instituciones, que transfiere el conocimiento y la experiencia existente entre los creadores de conocimiento científico, de modo que pueda ser utilizado como un recurso disponible para otros y sea sostenible en el tiempo (Nogueira et al., 2019).

Los administradores forestales necesitan acceso a información científica específica sobre los impactos del cambio climático para adaptarse al cambio climático en sí (Winkler, 2016). Las evaluaciones de 
vulnerabilidad abordan esta necesidad y son comunes en una variedad de disciplinas y geografías; sin embargo, la práctica de la evaluación de la vulnerabilidad ha revelado desafíos que merecen un examen más detenido en un contexto específico (Obermeister, 2017).

El informe especial del IPCC (2014) señala que el Perú es uno de los países de América Latina que se verá mayormente afectado por las consecuencias de la variabilidad del clima a escala estacional e interanual, ya que la mayoría de su producción depende de los extensos ecosistemas naturales con que cuenta. Con los conocimientos actuales, los datos disponibles y los niveles de incertidumbre que se enfrentan en el Perú, es aún muy complicado aproximar estimaciones más o menos precisas sobre lo que ocurriría en los diferentes tipos de bosque de la costa, sierra y selva. (Organización de las Naciones Unidas para la Agricultura y la Alimentación, 2016, p. 19)

La vulnerabilidad del Perú no sólo está relacionada a factores estructurales como la pobreza e inequidad, sino a los impactos operados en ecosistemas de importancia global como la Amazonía y los glaciares, responsables de la provisión y mantenimiento de la biodiversidad y los recursos hídricos (Ministerio del Ambiente, 2016). Los ecosistemas forestales y la diversidad biológica tienen una gran dependencia del clima; por tanto, su alta exposición y sensibilidad los hacen más vulnerables a los efectos del cambio climático. (Organización de las Naciones Unidas para la Agricultura y la Alimentación, 2016, p. 20).

Por ser los bosques tropicales fuente de bienes y servicios de gran importancia para los habitantes del planeta y particularmente para las comunidades que los habitan y que dependen de ellos, los cambios en los patrones del clima y la alteración de los ecosistemas hacen a estas poblaciones más vulnerables socioeconómicamente al cambio climático (Organización de las Naciones Unidas para la Agricultura y la Alimentación, 2016, p. 20). Dado que la vulnerabilidad es un proceso multidimensional en lugar de un estado inmutable, es más probable que un enfoque dinámico basado en ecosistemas con mayores índices de vulnerabilidad mejore los esfuerzos de planificación de mitigación y adaptación al cambio climático (Thomas et al., 2019).

En cada una de sus ocho áreas de evaluación, un panel de expertos locales aplicaron EVAA a ocho regiones del Medio Oeste y Noreste de Estados Unidos, en un total de 252 millones de acres (Brandt et al., 2017), determinaron la vulnerabilidad potencial de los ecosistemas forestales al cambio climático durante el próximo siglo. Desarrollaron calificaciones de vulnerabilidad e incertidumbre para los tipos de comunidades forestales en cada área de evaluación. La vulnerabilidad de los tipos de bosques individuales al cambio climático según la región debido a las diferencias regionales en cómo se espera que el cambio climático afecte a los impulsores del sistema, los factores de estrés y las especies dominantes y la capacidad de adaptación de una comunidad forestal (Brandt et al., 2017). Los resultados de dichas evaluaciones que utilizaron EVAA se continúan aplicando con éxito a las decisiones de manejo forestal en el Medio Oeste y el Noreste por administradores forestales no gubernamentales, privados y gubernamentales.

\section{Factibilidad de un escenario de confluencia}

Al aumentar la disposición de conocimiento y de competencias, las instituciones amplían sus oportunidades innovadoras, lo cual es posible, por ejemplo, mediante esfuerzos para incrementar la comprensión científica y tecnológica. De este modo, se esfuerzan por realizar actividades de investigación; y, en el ansia de crecer, crean alianzas estratégicas con otras instituciones ampliando su capacidad de aprendizaje. En este sentido, la integración de la academia es un objeto que merece atención por parte de quienes formulan políticas públicas, entendiendo que sus competencias científicas y tecnológicas tienden a ser importantes delimitadores de la eficiencia en la interacción con la sociedad y sus conflictos. Esta interacción puede justificarse dado que reduce las incertidumbres intrínsecas al proceso de innovación, diluyendo los riesgos relacionados a las actividades innovadoras. (Caliari and Chiarini, 2018) 
Estados Unidos tiene muy presente estos conceptos, es así como, su agencia gubernamental de manejo forestal, el Servicio Forestal, está evolucionando y seguirá siendo importante a medida que se presenten cada vez más oportunidades para la aplicación de evaluaciones de vulnerabilidad en la toma de decisiones de adaptación al cambio climático. Como una gran agencia gubernamental que administra una gran cantidad de tierras forestales para múltiples propósitos diferentes y, a veces, contrapuestos, esta agencia norteamericana ofrece un caso útil para examinar la práctica de las evaluaciones de vulnerabilidad y sus perspectivas para apoyar la adaptación. Si bien no todos sus hallazgos específicos se aplican a la ordenación forestal, los temas generales que se destacan son las asociaciones entre ciencia y ordenación como foco de entendimiento para las dimensiones científicas y políticas de la adaptación, resultando como instructivos en todos los ámbitos y gama de contextos de su ordenación forestal (Timberlake and Schultz, 2019).

Otros ejemplos en el exterior, el caso particular de los pequeños 'Estados insulares en Desarrollo del Caribe', que son vulnerables a los impactos del cambio climático incluido el aumento del nivel del mar, las especies invasoras, la acidificación de los océanos, los cambios en los patrones de lluvia, el aumento de las temperaturas y los regímenes de amenazas cambiantes, incluidos huracanes, inundaciones y sequías. Dada su alta dependencia de recursos naturales para subsistir en el proceso de adaptación al cambio climático, fue necesaria la aplicación de un enfoque centrado en los ecosistemas y su interacción con las personas. De esta manera, se destacaron los enfoques de adaptación basados en conocimiento científico en ecosistemas como una herramienta para abordar los impactos del cambio climático en sus políticas (Mercer et al., 2012). Estos dos casos reconocieron la importancia de distinguir a los ecosistemas forestales como fuentes de conocimiento científico, e integrar la producción de dicho conocimiento al desarrollo de proyectos de adaptación al cambio climático enmarcados en su legislación.

En el modelo de Estonia, no se visualizan vínculos específicos o directos con los resultados científicos forestales en sus procesos de formulación de políticas, sin embargo, es notoria la participación de investigadores en bosques. La Ley Forestal de Estonia (Riigikogu, 2019) per se, no tiene referencias directas a párrafos con base científica. No obstante, hay algunos aspectos o puntos de dicha legislación que están influenciados por los resultados de la investigación científica. Por ejemplo, en su Ley de Bosques (Riigikogu, 2019) se precisa que, en caso de tala rasa, los árboles de retención deben dejarse en el área con un mínimo de $5 \mathrm{~m}^{3} \mathrm{ha}^{-1}$. También hay puntos en su legislación cuestionados por sus regulaciones, por ejemplo, la de establecer edades de rotación específicas en especies forestales, donde en la investigación científica se cuestiona la necesidad de dichas regulaciones (Korjus et al., 2011). Este suceso, según lo explicaron Päivinen and Käär (2017), parece que responde principalmente a cuestiones políticas, el qué resultados de investigaciones se utilizan como adecuados o aplicables en su legislación.

Por otro lado, Finlandia suscita una problemática distinta a la anterior respecto a su marco legal forestal. Su legislación actual incluye medios de protección de la biodiversidad que se remontan a la década de 1990, según los cuales las leyes blandas y las medidas de protección voluntarias todavía reciben un gran énfasis en su política forestal (Yanagi and Nakamura, 2020). Esto ha sido insuficiente para detener el declive de su biodiversidad durante los últimos veinte años, tornándose más crítica debido a la creciente tala (Yanagi and Nakamura, 2020).

La descentralización ha sido el proceso de reforma más notable que se ha producido en los arreglos institucionales y el marco legal en Italia, un país de contrastes y contradicciones con recursos forestales atendidos, pero con una gama limitada de redes de conocimiento incapaces de hacer que el sector sea más moderno y dinámico (Secco et al., 2017). Italia pareciera estar experimentando varios de los problemas recurrentes destacados para la descentralización, incluidas las dificultades para crear las condiciones para generar iniciativas de gobernanza basadas en redes de conocimiento, que faciliten el acople y encaminamiento de sus objetivos en políticas forestales que hagan frente al cambio climático. 
Así, tras analizar los enfoques científicos sobre la importancia del derecho internacional y las organizaciones internacionales en la solución de problemas ambientales sobre la base de actos jurídicos internacionales, disposiciones teóricas de la ciencia jurídica internacional y materiales sobre los problemas de conservación ambiental, en nuestro país, el Estado reconoce que la extensión de los bosques y otros ecosistemas de vegetación silvestre se vienen reduciendo o degradando a lo largo del territorio, debido a una negativa combinación de factores económicos, sociales e institucionales (SERFOR 2020a). Estos procesos ponen en riesgo la capacidad de respuesta a las graves amenazas del cambio climático. (Ministerio de Agricultura y Riego, 2017)

'La Política Nacional del Ambiente (PNFFS) resalta la importancia de los bosques en el Perú, lo que incluye su papel en la mitigación y adaptación al cambio climático' (Ministerio de Agricultura y Riego, 2017). Por otra parte, la 'Ley Orgánica para el Aprovechamiento Sostenible de los Recursos Naturales'; precisa cómo el manejo racional toma en cuenta la capacidad de renovación de estos recursos. (Ministerio del Ambiente, 2017)

Por otro lado, ninguno de los principios de la Ley Orgánica para el Aprovechamiento Sostenible de los Recursos Naturales hace referencia al empleo de dichos recursos con fines de adaptación al cambio climático, tampoco sugiere su aprovechamiento para actividades de índole científica que conlleven a ese propósito.

Dentro de las Orientaciones establecidas en la PNFFS, la número (9) establece que el Estado debe alentar y promover la investigación y el desarrollo tecnológico, la gestión de los conocimientos científicos y tradicionales, la innovación, la extensión y la educación, como soportes estratégicos para impulsar el desarrollo forestal y de fauna silvestre sostenible (Ministerio de Agricultura y Riego, 2017). Empero, ninguna de estas orientaciones recoge o promueve la investigación y el desarrollo tecnológico, ni la gestión de los conocimientos científicos con fines de mitigación o adaptación al cambio climático.

El objetivo $\mathrm{N}^{\circ}$ (2) de la PNFFS refiere asegurar la conservación y el aprovechamiento sostenible de los bienes y servicios de los ecosistemas forestales fomentando una gestión integrada; así como la información oportuna, transparente y veraz para la toma de decisiones (Ministerio de Agricultura y Riego, 2017). A pesar de que este último renglón especifica en su texto que se respete el ordenamiento forestal, la seguridad sobre los derechos adquiridos, el monitoreo, la supervisión y la fiscalización; podría entenderse también, que suponga el uso de herramientas tecnológicas y científicas para la compresión de la temática asociada a los cambios del clima y sus impactos. De ser así, sería lo ideal según se plantea en este estudio.

En el marco de la implementación del Sistema Nacional de Información Forestal y de Fauna Silvestre (SNIFFS), que contiene seis módulos y cuatro componentes determinados por el (SERFOR 2020b), podrían enmarcase la propuesta de investigación, siendo de particular importancia el Módulo de Inventario y el Módulo de Gestión del conocimiento. El primero porque proveerá información de inventarios y evaluación de recursos para la toma de decisiones en el manejo forestal y, el segundo porque contendrá información relacionada al seguimiento y evaluación de la PNFFS - en actual actualización -, generando valor agregado de la información proveniente del resto de módulos SNIFFS, pudiendo visualizarse también la contención de un repositorio de publicaciones. Esta última podría relacionarse a el Mapa Nacional de Ecosistemas (Grupo de Trabajo para el Mapa Nacional de Ecosistemas, 2018b), que es un "instrumento que contribuye a la gestión del territorio y monitoreo de los ecosistemas y sus componentes, en particular la diversidad biológica, recursos naturales renovables y servicios ecosistémicos" (p. 13).

Finalmente, este escenario de confluencia responde además a lo previsto en la Ley $\mathrm{N}^{\circ} 30754$ Ley Marco sobre Cambio Climático (Ministerio del Ambiente, 2018), que en su artículo 19 habla sobre la generación y gestión de conocimiento científico sobre cambio climático mediante el desarrollo de la tecnología y la innovación por entidades públicas competentes en investigación, adscritas a las autoridades sectoriales. En 
el artículo 17, "el Ministerio del Ambiente es responsable del monitoreo y evaluación de la reducción de emisiones de deforestación y degradación de los bosques". Es así que, en el artículo 15 de la misma, "el Estado, en sus tres niveles de gobierno, de manera articulada y participativa, adopta las medidas de adaptación y aprovechamiento de oportunidades frente al cambio climático", priorizando la prevención y gestión de riesgos climáticos.

\section{DISCUSIÓN}

Este análisis de los cambios en los servicios de los ecosistemas forestales como fuentes de conocimiento científico identificó patrones generales en la forma en que los países han adaptado su legislación a los nuevos desafíos que trae consigo el cambio climático en las últimas décadas. La estimación del valor de los servicios ambientales ha representado un elemento crucial en el proceso de toma de decisiones sobre los programas y las políticas ambientales por desarrollar, pasando de políticas cuyas regulaciones ambientales respondían a flujos de comercio demarcando una irrompible conexión entre recursos naturales y economía internacional (Quijandría, 1993), a un enfoque de fijación de estándares que en términos de costos reducirían los efectos del cambio climático (Pasco-Font and Montoya, 1993).

La determinación del valor de los bienes ambientales ha representado diferentes enfoques en respuesta a los imperativos de las políticas económicas a lo largo de las últimas décadas, estas parecían responder a ecuaciones de valorización cuyas variables eran netamente económicas que, posteriormente adhirieron variables un poco más comprometidas con el bienestar ambiental. Pero ¿cómo se define hoy ese bienestar?, ¿cómo se traduce en la ecuación de valorización? Definidamente se está frente a una temática transversal, por cómo lo entienden Roa et al. (2004), se trataría de un escenario en el que el valor de los bienes ambientales está inferido por algún modelo de elección y de comportamiento particular. Precisar lo que hoy se entiende por bienestar continúa representando vacilación, sin embargo, por cómo se ha explicado, las variables de vulnerabilidad y adaptación sugieren un enfoque un tanto, si no mucho más sostenible, en comparación a los que antes regían el valor de los bienes ambientales.

Esta incertidumbre constitutiva de los procesos de innovación en políticas ambientales parece reducirse en cuanto se anexa el conocimiento científico a las directrices de quienes formulan políticas públicas, entendiendo que este puede repercutir en qué tan eficiente resulta la interacción de la sociedad y los conflictos ambientales que sugiere el cambio climático (Caliari and Chiarini, 2018). Estonia por su parte contempla el empleo de conocimiento científico en la regulación de su sistema legal forestal (Riigikogu, 2019), que por un lado, ha coadyuvado a proteger su patrimonio forestal y a fortalecer acciones frente al cambio climático, lo que a su vez, ha producido un contraste entre diferentes opiniones de grupos de científicos (Korjus et al., 2011), esto podría deberse a la escasa sistematización de la información científica que producen, que conlleva a una divulgación incompleta de la misma, siendo que la participación de estos científicos en la toma de decisiones se da desde la intervención de particulares. Por otro lado, Finlandia, pese a poseer un mejor estado de biodiversidad respecto a los demás países de la Unión Europea (Yanagi and Nakamura, 2020), urge de implementar mejoras significativas en su gobernanza forestal que partan del conocimiento científico y no de normas estandarizadas internacionalmente cuya vigencia data de tres décadas atrás. Esto ejemplifica una ruta de trabajo inevitable si es que se quiere lograr el ansiado desarrollo sostenible.

Una vez superados los inconvenientes presentados en los dos casos anteriores, ¿estarían estas naciones preparadas para enfrentar nuevos desafíos que les presente el cambio climático? Italia, que a pesar de tener cubierta la generación de conocimiento científico que sirve de base para el desarrollo y regularización de sus políticas forestales, denota una problemática, posiblemente consecuente a las anteriores, en la que se advierte un vacío de redes de conocimiento, que producto de sus políticas de descentralización, han conllevado a la fragmentación de sus sistemas de información en cuanto a adaptación al cambio climático. 
Entonces, ¿cómo el proceso legislativo y de formulación de políticas facilita la interacción de la ciencia y el derecho ambiental? El estudio de Čavoški (2020) examina cómo la Comisión Europea crea esta interacción entre ciencia y derecho en la formulación de políticas ambientales e identifica cómo incorpora diferentes voces y partes interesadas en esta área política. Es así que, la Comisión puede considerarse un vehículo de coproducción de ciencia y derecho en la política medioambiental de la Unión Europea mediante la construcción de identidades sólidas de expertos y la puesta en marcha de procesos e instrumentos institucionales, y la creación de un discurso entre científicos y abogados que conducen a resultados de coproducción. Al facilitar activamente la coproducción, la Comisión sustenta el proceso legislativo y de formulación de políticas con sus valores institucionales.

¿Por qué se sugiere que la implementación de los módulos del SNIFES y su respaldo en una actualizada PNFFS son de vital importancia para resolver la problemática planteada en el Perú? Aquí se desarrollan diversas investigaciones orientadas a resolver problemáticas propias de lo que sugiere el cambio climático, que emplean el aprovechamiento de los recursos forestales para las mismas. Podría resultar trascendental para la consecución de dichas prácticas, la creación del Plan Nacional de Investigación Forestal y Fauna Silvestre, que pretende ser un instrumento de planificación para la gestión forestal y de fauna silvestre que permitirá impulsar el sector articulándose al Plan Estratégico de Desarrollo Nacional entre otros, pues, de acuerdo con ley se enmarca en la PNFFS, acorde con la versión de altos funcionarios del Ministerio del Ambiente (SERFOR, 2020a).

En cuanto a la sistematización de información científica en el Perú, en su cuarto eje temático 'Herramientas para la toma de decisiones', la Agenda nacional de investigación científica en cambio climático 2010-2021 (Ministerio del Ambiente, 2010), comprende cinco líneas de investigación. Lamentablemente, ninguna de estas líneas ni sus indicadores responden a las preguntas de investigación de este estudio, siendo la más cercana, la del Diseño y desarrollo de sistemas locales de alerta temprana para riesgos de desastre asociados al cambio climático en áreas sensibles del territorio nacional, esto porque las demás líneas no se ajustan a un carácter preventivo. Por su parte, los indicadores para el desarrollo de estas líneas solo enumeran el recojo de información y su asimilación, mas no ahondan en sus objetivos ni aúnen sus estrategias.

\section{CONCLUSIÓN}

Partes importantes del proceso adaptativo residen en la práctica y la innovación en el bosque, en lugar de enfoques jerárquicos y dirigidos por las leyes, pero la realidad no presenta una dicotomía simple entre el manejo forestal determinista y reduccionista y los enfoques ecosistémicos indeterministas y adaptativos. Es necesario prestar más atención a las realidades de los profesionales y su contribución al conocimiento. En el marco de este estudio, se reflexionó sobre la estrategia de hacer frente el cambio climático, mediante el desarrollo de conocimiento científico basado en ecosistemas forestales. Esto coadyuvaría a la correcta toma de dediciones y a la gestión sostenible del conocimiento científico en adaptación al cambio climático, cumpliendo los imperativos y compromisos normativos expuestos en anteriores párrafos.

El análisis ha ilustrado cómo los diferentes enfoques de gobernanza forestal frente al cambio climático existen y se desarrollan de diferentes maneras a nivel internacional, enfatizando el papel del estado en cómo se direccionan y bajo qué supuestos ha sido desarrollado su marco legal en respuesta a estas políticas.

Es posible que las diferentes herramientas e instrumentos desarrollados en los diversos documentos nacionales que pretenden hacer frente al cambio climático no respondan a iniciativas basadas en conocimiento científico propio y que no se centren en un repositorio de resultados de investigaciones que permitan facilitar el proceso de definición de estas estrategias, y cómo estos podrían ser la causa de que no se haya evidenciado hasta ahora una operativización que brinde resultados tangibles. 
El enfoque analizado proporciona nuevos conocimientos y permite analizar la dinámica y capacidad de respuesta de los sistemas de política forestal, integrando conceptos de adaptación y mitigación. De ahí que demuestre el valor de la vulnerabilidad de un ecosistema forestal como herramienta de análisis de políticas de cambio, además de los beneficios instantáneos que devienen de la sistematización de información científica.

Finalmente, avocarse a las evaluaciones de vulnerabilidad en el contexto nacional para hacer frente al cambio climático, basadas en conocimiento científico confiable, resulta valioso no solo por abordar temas poco trabajados sino por el enfoque multidisciplinario que les da a los ecosistemas forestales y por las reflexiones que brinda en torno a aspectos que serán cada vez más preocupantes para la realidad peruana y global contemporánea.

\section{AGRADECIMIENTOS}

Esta investigación se realizó en el marco del "Taller de Elaboración de Publicaciones Científicas" impulsada por la Dra. Elena Lon Kan Prado, Vicepresidenta de Investigación de la Universidad Nacional Tecnológica de Lima Sur.

\section{REFERENCIAS}

Abrahams, D., and Carr, E. R. (2017). Understanding the Connections Between Climate Change and Conflict: Contributions From Geography and Political Ecology. In Current Climate Change Reports (Vol. 3, Issue 4, pp. 233-242). Current Climate Change Reports. https://doi.org/10.1007/s40641-017-0080-Z

Barcelo-Valenzuela, M., Sanchez-Schmitz, G., Perez-Soltero, A., Martín Rubio, F., and Palma, J. (2008). Defining the problem: Key element for the success of knowledge management. Knowledge Management Research and Practice, 6(4), 322-333. https://doi.org/10.1057/kmrp.2008.22

Beier, P., Hansen, L. J., Helbrecht, L., and Behar, D. (2017). A How-to Guide for Coproduction of Actionable Science. Conservation Letters, 10(3), 288-296. https://doi.org/10.1111/conl.12300

Brandt, L. A., Butler, P. R., Handler, S. D., Janowiak, M. K., Shannon, P. D., and Swanston, C. W. (2017). Integrating science and management to assess forest ecosystem vulnerability to climate change. Journal of Forestry, 115(3), 212-221. https://doi.org/10.5849/jof.15-147

Bustamante, D. (2009). El Diseño de la Investigación Jurídica (pp. 1-34). Editorial Universidad del Valle. Cadilhac, L., Torres, R., Calles, J., Vanacker, V., and Calderón, E. (2017). Desafíos para la investigación sobre el cambio climático en Ecuador. Neotropical Biodiversity, 3(1), 168-181. https://doi.org/10.1080/23766808.2017.1328247

Caliari, T., and Chiarini, T. (2018). Análisis de los grupos de investigación de las áreas científicas con mayor aplicabilidad productiva en el Brasil: competencias e interacciones con las empresas. Apuntes: Revista de Ciencias Sociales, 45(82), 71-98. https://doi.org/10.21678/apuntes.82.864

Castells-Quintana, D., Lopez-Uribe, M. del P., and McDermott, T. K. J. (2018). Adaptation to climate change: A review through a development economics lens. World Development, 104, 183-196. https://doi.org/10.1016/j.worlddev.2017.11.016

Čavoški, A. (2020). Science and Law in Environmental Law and Policy: The Case of the European Commission. Transnational Environmental Law, 9(2), 263-295. 
https://doi.org/10.1017/s2047102520000151

Dalmazzo-Bermejo, E., Valenzuela-Klagges, B., and Espinoza-Brito, L. (2017). Producción de energía renovable no tradicional en América Latina: economía y políticas públicas. Apuntes: Revista de Ciencias Sociales, 44(81), 67-87. https://doi.org/10.21678/apuntes.81.806

Dreyfus, M. (2015). Adaptation to Climate Change in Cities. Handbook of Climate Change Adaptation, February, 687-705. https://doi.org/10.1007/978-3-642-38670-1_67

Flores, P. (2008). Las posibilidades para desarrollar el proceso de EAE en el Perú. Apuntes: Revista de Ciencias Sociales, 20. https://doi.org/10.21678/apuntes.56/57.553

Grupo de Trabajo Multisectorial. (2018). Informe Final sobre la información técnica para la implementación de las Contribuciones Nacionalmente Determinadas. Ministerio del Ambiente.

Grupo de Trabajo para el Mapa Nacional de Ecosistemas. (2018). Mapa Nacional de Ecosistemas. In Memoria Descriptiva (p. 60). Portal Institucional del Ministerio del Ambiente.

Hernández Sampieri, R., and Mendoza Torres, C. P. (2018). Metodología de la investigación: las tres rutas cuantitativa, cualitativa y mixta. In Mc Graw Hill (Vol. 1, Issue Mexico).

IPCC. (2014). Cambio climático 2014. In Equipo principal de redacción (Ed.), OMM; PNUMA (5th ed.). Grupo Intergubernamental de Expertos sobre el Cambio Climático. https://www.ipcc.ch/site/assets/uploads/2018/02/SYR_AR5_FINAL_full_es.pdf

Kámiche, J., and Cárdenas García Santillán, M. K. (2008). ¿Qué tan "verdes” son los gobiernos locales y regionales? Un análisis a partir de su interés en la inversión pública ambiental en el Perú. Apuntes: Revista de Ciencias Sociales, 63, 27-77. https://doi.org/10.21678/apuntes.63.576

Korjus, H., Põllumäe, P., and Rool, S. (2011). Männi-, kuuse- ja kasepuistute majandamise tasuvus lühikese raieringi korral. Forestry Studies, 54(1978), 28-36. https://doi.org/10.2478/v10132-011-0093-7

Lossio, J. (2015). CAREY, Mark, 2014, Glaciares, cambio climático y desastres naturales. Ciencia y sociedad en el Perú, Lima, IEP e IFEA. 343 pp. Apuntes: Revista de Ciencias Sociales, 42(77), 190-192. https://doi.org/10.21678/apuntes.77.744

Mercer, J., Kelman, I., Alfthan, B., and Kurvits, T. (2012). Ecosystem-based adaptation to climate change in caribbean small island developing states: Integrating local and external knowledge. Sustainability, 4(8), 1908-1932. https://doi.org/10.3390/su4081908

Ministerio de Agricultura y Riego. (2017). Política nacional forestal y de fauna silvestre (1st ed.). Servicio $\begin{array}{lllll}\text { Nacional Forestal } & y & \text { de } & \text { Fauna } & \text { Silvestre. }\end{array}$ https://www.minagri.gob.pe/portal/download/pdf/especiales/politica-nacional.pdf

Ministerio del Ambiente. (2010). AGENDA NACIONAL DE INVESTIGACIÓN CIENTÍFICA EN CAMBIO CLIMÁTICO 2010-2021- AIC-CC - (Ministerio del Ambiente and Consejo Nacional de ciencia Tecnología e innovación Tecnológica (eds.)). Sistema Naciona de Información Ambiental.

Ministerio del Ambiente. (2015). Estrategia Nacional ante el Cambio Climático. In Sistema Nacional de Información Ambiental (Primera ed). Ministerio del Ambiente. 
Ministerio del Ambiente. (2016). El Perú y el Cambio Climático. Tercera Comunicación Nacional del Perú. In Convención Marco de las Naciones Unidas sobre Cambio Climático (Tercera ed). Sistema Nacional de Información Ambiental.

Ministerio del Ambiente. (2017). Ley Orgánica para el aprovechamiento sostenible de los recursos naturales LEY No 26821 (p. 55). Diario Oficial El Peruano.

Ministerio del Ambiente. (2018). Ley No 30754. Ley Marco sobre Cambio Climático. In Diario Oficial "El Peruano" (Issues 1638161-1). Sistema Nacional de Información Ambiental.

Montero, L. (1995). La economía cocalera en el Perú: implicancias sobre las migraciones y el medio ambiente. Apuntes: Revista de Ciencias Sociales, 074, 105-122. https://doi.org/10.21678/apuntes.36.417

Nogueira, D. M., Esther, Y., Nogueira, M., Vega, V., and Assafiri, Y. El. (2019). Título : Inventario de conocimiento en los Observatorio Científicos: herramienta de mejora de proceso . April. https://doi.org/10.13140/RG.2.2.20803.04649

Obermeister, N. (2017). From dichotomy to duality: Addressing interdisciplinary epistemological barriers to inclusive knowledge governance in global environmental assessments. Environmental Science and Policy, 68, 80-86. https://doi.org/10.1016/j.envsci.2016.11.010

Organización de las Naciones Unidas para la Agricultura y la Alimentación. (2016). Los bosques y el cambio climatico en el Peru. In FAO (14th ed.). Organización de las Naciones Unidas para la Alimentación y la Agricultura.

Ortiz, C., and Ortega, P. (2016). Retomando fundamentos y paradigmas para el tránsito de la crisis ambiental hacia sociedades sustentables. Sociedad y Ambiente, 10, 113-131. https://doi.org/10.31840/sya.v0i10.1655

Päivinen, R., and Käär, L. (2017). Tools for improving science-practice interaction in forestry (Issue 20).

Pasco-Font, A., and Montoya, A. (1993). El empleo de incentivos económicos de la protección del medio ambiente. Apuntes: Revista de Ciencias Sociales, 32, 21-31. https://doi.org/10.21678/apuntes.32.366

Quijandría, Á. (1993). El comercio internacional y el medio ambiente. Apuntes: Revista de Ciencias Sociales, 33, 49-59. https://doi.org/10.21678/apuntes.33.379

Quintero, J., Ancízar, R., and Munévar, F. (2008). Semilleros de investigación: una estrategia para la formación de investigadores. Educación y Educadores, 11(1), 31-42.

Riigikogu. (2019). Riggi Teataja. Terviktekst.

Roa, Z., Rodríguez, G., Rosales Álvarez, R., and Requier-Desjardins, D. (2004). Estimación de la disposición a pagar por panela ecológica desde el enfoque del consumo sostenible. Apuntes: Revista de Ciencias Sociales, 54, 59-87. https://doi.org/10.21678/apuntes.54.538

Scarano, F. R. (2017). Ecosystem-based adaptation to climate change: concept, scalability and a role for conservation science. Perspectives in Ecology and Conservation, 15(2), 65-73. https://doi.org/10.1016/j.pecon.2017.05.003

Secco, L., Favero, M., Masiero, M., and Pettenella, D. M. (2017). Failures of political decentralization in promoting network governance in the forest sector: Observations from Italy. Land Use Policy, 62, 79-100. 
https://doi.org/10.1016/j.landusepol.2016.11.013

SERFOR. (2020a). Plan Nacional De Investigación Forestal y de Fauna Silvestre 2020-2030 (1st ed.). Servicio Nacional Forestal y de Fauna Silvestre. https://bit.ly/37tR92S

SERFOR. (2020b). Sistema Nacional de Información Forestal y Fauna Silvestre. Ministerio de Agricultura y Riego

Strauss, A., and Corbin, J. (2002). Bases de la investigación cualitativa: técnicas y procedimientos para desarrollar la teoría fundamentada.

Sung, E., and Kim, Y. (2016). Adaptation to Climate Change: Decision Making. In Sustainable Water Resources Planning and Management Under Climate Change (pp. 189-221). Springer. https://doi.org/10.1007/978-981-10-2051-3

Thomas, K., Hardy, R. D., Lazrus, H., Mendez, M., Orlove, B., Rivera-Collazo, I., Roberts, J. T., Rockman, M., Warner, B. P., and Winthrop, R. (2019). Explaining differential vulnerability to climate change: A social science review. Wiley Interdisciplinary Reviews: Climate Change, 10(2), 1-18. https://doi.org/10.1002/wcc.565

Timberlake, T. J., and Schultz, C. A. (2019). Climate change vulnerability assessment for forest management: The case of the U.S. Forest Service. Forests, 10(11). https://doi.org/10.3390/f10111030

Ulibarri, N. (2018). Collaborative model development increases trust in and use of scientific information in environmental decision-making. Environmental Science and Policy, 82(January), 136-142. https://doi.org/10.1016/j.envsci.2018.01.022

Winkler, J. A. (2016). Embracing Complexity and Uncertainty. Annals of the American Association of Geographers, 106(6), 1418-1433. https://doi.org/10.1080/24694452.2016.1207973

Wise, C. (2005). De la apatía a la vigilancia: la política del desarrollo energético en el Perú. Apuntes: Revista de Ciencias Sociales, 37. https://doi.org/10.21678/apuntes.56/57.547

Yanagi, K., and Nakamura, A. (2020). Towards a Low/Zero Carbon Society for the Asia-Pacific Region: Policy and Legal Development for Carbon Capture and Storage (CCS) in Japan. In Sustainability and Law. https://doi.org/10.1007/978-3-030-42630-9_29

Yousefpour, R., Temperli, C., Jacobsen, J. B., Thorsen, B. J., Meilby, H., Lexer, M. J., Lindner, M., Bugmann, H., Borges, J. G., Palma, J. H. N., Ray, D., Zimmermann, N. E., Delzon, S., Kremer, A., Kramer, K., Reyer, C. P. O., Lasch-Born, P., Garcia-Gonzalo, J., and Hanewinkel, M. (2017). A framework for modeling adaptive forest management and decision making under climate change. Ecology and Society, 22(4). https://doi.org/10.5751/ES-09614-220440

Zhang, M., Liu, Z., and Van Dijk, M. P. (2019). Measuring urban vulnerability to climate change using an integrated approach, assessing climate risks in Beijing. PeerJ, 2019(5), 1-19. https://doi.org/10.7717/peerj.7018 